\title{
Ground state and excited states of a trapped dilute condensed Bose gas
}

\author{
Alexander L. Fetter \\ Departments of Physics and Applied Physics, Stanford University \\ Stanford, California 94305-4060, USA \\ I review recent theoretical treatments of a dilute interacting condensed Bose gas in a trap. Bogoliubov's classic \\ results for a uniform condensate are generalized to include the effect of a trap, using the Gross-Pitaevskii \\ formalism (for the condensate) and the Bogoliubov equations (for the linearized small-amplitude excitations \\ of the condensate). Several recent theoretical studies are discussed along with some open questions.*
}

\section{INTRODUCTION}

Although ${ }^{4} \mathrm{He}$ was liquified in 1908 and the anomalous peak in the heat capacity at $T_{\lambda} \approx$ $2.2 \mathrm{~K}$ was discovered in 1932, its remarkable lowtemperature superfluidity was observed only in 1938 by Kapitza and by Allen and Misener [1, 2]. Soon afterward, Landau's bold 1941 theory of superfluidity of He II (including both the two-fluid hydrodynamics and the quasiparticle picture of phonons and rotons) 3, 2, 4] was brilliantly confirmed by Andronikashvili, Kapitza, and Peshkov [1, 2]. In 1947, Bogoliubov [5, 6, 7] proposed the first microscopic description of superfluidity in a uniform weakly interacting Bose gas and (with acknowledgment to Landau) suggested the correct generalization to a dilute gas with strong repulsive interactions. This work is summarized in Sec. 2; it has since served as the basis for studies of a nonuniform dilute Bose gas [8, 9, 10].

The last year has seen the exciting development of wholly new Bose condensates consisting of dilute alkali atoms 11, 12, 13, 14. At this conference, the F. London award has been presented to Cornell and Wieman, with comprehensive invited talks by Wieman, Ketterle, and Jin. The present work seeks to build on these experimental achievements by reviewing the theoretical situation for a dilute Bose gas at low temperatures, well below the onset of BoseEinstein condensation. The presence of the confining (typically harmonic) trap introduces essential new features that are summarized in Secs. 3 and 4 (for the ground state and low-lying excited states, respectively). Section 5 considers various specific calculations that have already been performed, and Sec. 6 provides a partial list of open interesting questions.

\section{REVIEW OF BOGOLIUBOV THEORY}

Five decades ago, Bogoliubov [5, 7 proposed a simple and intuitive description of a uniform dilute Bose gas, focusing particularly on how the interacting ground state differs from that of an ideal Bose gas [1]. Since the repulsive interactions favor a uniform configuration, Bogoliubov used periodic boundary conditions to ensure that the unperturbed ideal-gas single-particle ground state is the uniform Fourier component with $\mathbf{k}=\mathbf{0}$. This choice is necessary because, for other boundary conditions, the unperturbed ground state would not be even approximately uniform; in the thermodynamic limit of $N$ particles in a volume $V$ (keeping the particle density $n \equiv N / V$ fixed), the introduction of weak repulsive interactions produces a dramatic change in any nonuniform equilibrium density profile (for example, an ideal Bose gas in a box with rigid walls has a density proportional to products of squared real trigonometric functions, whereas the interacting density is essentially uniform except very near the walls).

An ideal Bose gas at zero temperature has all particles in the condensate, so that the number $N_{0}$ of particles with zero momentum is just the total $N$; for an interacting system, however, $N_{0}$ is smaller than $N$ (in lowest-order perturbation theory, two particles can scatter out of the condensate and occupy the many zero-total-momentum states with separate momenta $\mathbf{k}$ and $\mathbf{- k}$ ). For a dilute interacting Bose gas with a macroscopic condensate $\left(N_{0} \gg 1\right)$, Bogoliubov made the very simple observation that the

* Research supported in part by the National Science Foundation under Grant No. DMR 94-21888. 
commutator of the second-quantized condensate operators $\left[c_{0}, c_{0}^{\dagger}\right]=1$ is small compared to their separate effect on the ground state (which yields a factor of order $\sqrt{N_{0}} \gg 1$ ). Thus these condensate operators can be treated as simple numbers. In addition, Bogoliubov assumed that most of the particles remain in the condensate, with $N^{\prime} \equiv N-N_{0} \ll N$ so that the total depletion of the condensate remains small. This condition means that it is sufficient to keep only terms of second order in the noncondensate operators, and a linear (canonical) transformation can diagonalize the resulting approximate hamiltonian.

For plane-wave states $\left(\propto e^{i \mathbf{k} \cdot \mathbf{r}}\right)$ at $T=0 \mathrm{~K}$, a particle with wave vector $\mathbf{k}$ has two characteristic energies: the kinetic energy

$$
T_{k}=\hbar^{2} k^{2} / 2 m,
$$

and the mean "Hartree" interaction energy with the macroscopic condensate

$$
V_{H} \equiv n_{0} V_{0},
$$

where $V_{0}$ is the spatial integral of the interparticle potential (namely the $\mathbf{k}=\mathbf{0}$ component of the Fourier transform); note that $V_{H}$ is effectively a constant optical potential affecting the propagation through the medium. As proposed by Bogoliubov/Landau and proved subsequently by Lee, Huang, and Yang [5, 6], the correct generalization for a strong repulsive interparticle potential (whose Fourier transform diverges) is to replace $V_{0}$ by $4 \pi a \hbar^{2} / m$, where $a$ is the $s$-wave scattering length; in this case, the Hartree potential becomes $V_{H}=$ $4 \pi a \hbar^{2} n_{0} / m$.

One of Bogoliubov's central results is the energy eigenvalue for a plane wave:

$$
E_{k}=\sqrt{2 V_{H} T_{k}+T_{k}^{2}}
$$

Here, the first term under the square root is proportional to $k^{2}$ and the second to $k^{4}$, so that $E_{k}$ has the following limits

$$
E_{k} \approx \begin{cases}\sqrt{4 \pi a n_{0}} \hbar^{2} k / m, & \text { for } k \rightarrow 0 \\ \hbar^{2} k^{2} / 2 m, & \text { for } k \rightarrow \infty\end{cases}
$$

In particular, the long-wavelength dispersion relation is simply that of a sound wave (a phonon) with propagation speed $s \equiv \sqrt{4 \pi a n_{0}} \hbar / m$, given by the appropriate thermodynamic compressibility [6]. This characteristic linear long-wavelength spectrum reflects the presence of the Bose condensate (note that $\left.s^{2} \propto n_{0}\right)$ and differs qualitatively from that of a dilute Fermi gas (for comparison, a nucleon moving through a nucleus with wave vector $\mathbf{k}$ has an energy $\approx V_{H}+T_{k}$, which has a gap at $k=0$ ). For a uniform Bose gas, the transition between the phonon and free-particle spectrum occurs at a wavenumber

$$
k_{0}=\sqrt{8 \pi a n_{0}}=\sqrt{2} m s / \hbar
$$

obtained by setting $T_{k_{0}}=V_{H}$. The interactions must be repulsive $(a>0)$ to ensure that $s$ and $k_{0}$ are real; in addition, the interactions play a crucial physical role, for $k_{0}$ vanishes if $a \rightarrow 0$. As noted by Bogoliubov (based on Landau's quasiparticle picture), the critical velocity $v_{c}$ for the destruction of superfluidity is here simply the speed of sound, $s \propto \sqrt{a n_{0}}$, again emphasizing the need for interparticle repulsion (in particular, $v_{c}=0$ for a uniform ideal Bose gas).

In addition to the energy eigenvalue, Bogoliubov's canonical transformation determines the corresponding eigenstate, which involves a Bose "quasiparticle" created by the operator

$$
\gamma_{\mathbf{k}}^{\dagger} \equiv u_{k} c_{\mathbf{k}}^{\dagger}+v_{k} c_{-\mathbf{k}}
$$

This description looks very similar to the familiar (and later) BCS theory of superconductivity 15, but here the "coherence factors" satisfy the condition

$$
u_{k}^{2}-v_{k}^{2}=1
$$

to ensure that the quasiparticle operators obey Bose commutation relations. In the interacting ground state, Bogoliubov showed that the occupation number $N_{k}^{\prime}$ of the state with wave vector $\mathbf{k}$ is simply $v_{k}^{2}=\frac{1}{2}\left[E_{k}^{-1}\left(V_{H}+T_{k}\right)-1\right]$, with the limiting forms

$$
N_{k}^{\prime} \approx \begin{cases}k_{0} / \sqrt{8} k \gg 1, & \text { for } k \ll k_{0}, \\ k_{0}^{4} / 4 k^{4} \ll 1, & \text { for } k \gg k_{0} .\end{cases}
$$

The total fractional ground-state depletion of the condensate follows by summing over all nonzero plane waves:

$$
\frac{n^{\prime}}{n}=\frac{1}{n} \int \frac{d^{3} k}{8 \pi^{3}} v_{k}^{2}=\frac{8}{3} \sqrt{\frac{n_{0} a^{3}}{\pi}} .
$$

It is important to recall that this finite zerotemperature depletion arises solely from the presence of the repulsive interactions. At finite temperature, thermal fluctuations induce additional depletion of the condensate [10], analogous to that for an ideal Bose gas. Equation (9) shows that the Bogoliubov condition of small depletion $\left(N^{\prime} \ll N\right)$ thus requires 
$\sqrt{n_{0} a^{3}} \ll 1$; this depletion is nonanalytic in the scattering length $a$ and cannot be obtained with any finite order in perturbation theory (this is the principal advantage of the canonical transformation). A combination with Eq. (5) yields the alternative condition $k_{0} a \ll 1$ for a dilute Bose condensate.

An equivalent hydrodynamic description characterizes the same basic physics. In this case, the operator

$$
\rho_{\mathbf{k}}^{\dagger} \approx \sqrt{n_{0}}\left(c_{\mathbf{k}}^{\dagger}+c_{-\mathbf{k}}\right)
$$

creates a density fluctuation with wave vector $\mathbf{k}$; it has the same energy $E_{k}$ given in Eq. (3), even though it is a different linear combination than the quasiparticle operator in Eq. (6). It is easy to show that these "phonons" are longitudinal and hence irrotational; as discussed below, vorticity occurs only for condensates containing discrete vortex lines.

\section{NONUNIFORM CONDENSATE}

Gross and Pitaevskii [8, 9, 10] extended Bogoliubov's theory to describe a rectilinear vortex line in an otherwise uniform dilute Bose gas. The condensate density $n_{0}(\mathbf{r})$ is now spatially varying, and it convenient to introduce a "condensate wave function" $\Psi(\mathbf{r})$ whose squared absolute value is just the condensate density $|\Psi(\mathbf{r})|^{2}=n_{0}(\mathbf{r})$. Evidently, the total number of condensed particles is given by $N_{0}=$ $\int d^{3} r|\Psi(\mathbf{r})|^{2}$, thus fixing the normalization. In the present context of trapped alkali atoms, the GrossPitaevskii method must be generalized to include the trap potential $V(\mathbf{r})$; although the actual traps are anisotropic, the present treatment will consider only the simpler case of an isotropic harmonic trap with

$$
V(\mathbf{r})=\frac{1}{2} m \omega_{0}^{2} r^{2} .
$$

In contrast to the situation for a uniform condensate, there are now three separate contributions to the total energy: the kinetic energy

$$
T=-\frac{\hbar^{2} \nabla^{2}}{2 m}
$$

the spatially varying Hartree energy of a single particle with the nonuniform condensate

$$
V_{H}(\mathbf{r})=n_{0}(\mathbf{r}) V_{0}=\frac{4 \pi a \hbar^{2}}{m} n_{0}(\mathbf{r}),
$$

along with the trap potential $V(\mathbf{r})$. The condensate wave function satisfies a nonlinear Schrödinger (Gross-Pitaevskii) equation

$$
\left(T+V+V_{H}\right) \Psi=\mu \Psi
$$

where $\mu$ is the chemical potential (given at $T=0 \mathrm{~K}$ by $\mu=\partial E / \partial N)$. The nonlinearity arises from the Hartree potential, proportional to $|\Psi|^{2}$. Equation (14) is formally like the "Ginzburg-Pitaevskii" equation 16], but its interpretation is very different. Here, the Gross-Pitaevskii equation describes a nonuniform Bose condensate with small depletion at low temperature, whereas the Ginzburg-Pitaevskii equation is analogous to the Ginzburg-Landau theory of the second-order normal-superconducting phase transition [17 and thus holds only near the transition temperature, where the (thermal) depletion is necessarily large.

The trap potential introduces an additional length scale $d_{0}$ associated with the zero-point motion in the trap; setting $\hbar \omega_{0}=\hbar^{2} / m d_{0}^{2}$ yields the familiar oscillator length

$$
d_{0}=\sqrt{\hbar / m \omega_{0}} .
$$

For comparison, it is convenient to use the scattering length $a$ and the "coherence" or "healing" length

$$
\xi \equiv 1 / k_{0}=1 / \sqrt{8 \pi a n_{0}}=\hbar / \sqrt{2} m s
$$

that characterizes the distance over which the condensate wave function heals back to its asymptotic value when subjected to a local perturbation ( $\xi$ is analogous to the BCS or Ginzburg-Landau coherence length in a superconductor [17]).

In the case of trapped ${ }^{87} \mathrm{Rb} 11$, these parameters are $a \approx 10 \mathrm{~nm}$ and $d_{0} \approx$ a few $\mu \mathrm{m}$, so that $a \ll d_{0}$; for the typical particle density $n \approx 10^{20} \mathrm{~m}^{-3}$, the coherence length is $\xi \approx$ a few $\times 0.1 \mu \mathrm{m}$. If $\xi \gg d_{0}$, then the healing length is large compared to the trap dimension and the system is nearly ideal; in this case, the selfconsistent condensate wave function is the Gaussian ground state $\Psi_{G}$ of the harmonic oscillator (the corresponding condensate density $n_{0}=\left|\Psi_{G}\right|^{2}$ is also Gaussian).

For most experiments, however, the opposite condition $a \ll \xi \ll d_{0}$ applies, and the system is dilute and interacting rather than ideal, so that the interparticle repulsion expands the condensate significantly; in this limit, the kinetic energy operator has only a small effect on Eq. (14), leading to an algebraic equation

$$
\left(V+V_{H}-\mu\right) \Psi_{T F}=0 .
$$

The resulting "Thomas-Fermi" (TF) approximation for the condensate density [18, 19, 20] yields

$$
\frac{4 \pi a \hbar^{2}}{m} n_{0 T F}(\mathbf{r})=\mu-V(\mathbf{r})
$$


wherever the right-hand side is positive and zero elsewhere. In the present case of an isotropic harmonic potential, the TF condensate density has an inverted parabolic profile $n_{0 T F}(r) \propto\left(R^{2}-r^{2}\right) \theta(R-r)$, where $\theta$ denotes the unit positive step function, $R / d_{0} \approx$ $\left(15 \mathrm{Na} / d_{0}\right)^{1 / 5} \gg 1$ characterizes the "condensate radius" $R$, and $\mu \approx \frac{1}{2} \hbar \omega_{0} R^{2} / d_{0}^{2}$ is the chemical potential [20]. It is often preferable to define the dimensionless ratio $N a / d_{0}$, which is large (small) for dilute interacting (ideal) Bose gas. As an aside, bulk liquid ${ }^{4} \mathrm{He}$ is "dense," for it has $\xi \approx a$.

\section{SMALL-AMPLITUDE EXCITATIONS OF A NONUNIFORM CONDENSATE}

To treat the small-amplitude excited states in a trap, it is necessary to generalize the Bogoliubov quasiparticles to the case of a nonuniform condensate (this approach was originally developed for a vortex in a dilute Bose gas [9], but the inclusion of the trap potential is straightforward). Formally, introduce an operator $\phi^{\dagger}(\mathbf{r})$ that creates a noncondensate particle at $\mathbf{r}$ [it is simply the fundamental second-quantized field operator $\psi^{\dagger}(\mathbf{r})$ with the condensate operator and wave function $\Psi(\mathbf{r})$ removed]. This particle operator can be expressed as a linear combination of quasiparticle operators $\gamma_{j}^{\dagger}$ and $\gamma_{-j}$ [compare Eq. (6)], where the index $j$ denotes one of a set of complete normal modes and $-j$ denotes the time-reversed set of quantum numbers 21]. The corresponding expansion coefficients are the Bogoliubov amplitudes (analogous to wave functions) $u_{j}(\mathbf{r})$ and $v_{j}(\mathbf{r})$ that obey the coupled linear Bogoliubov eigenvalue equations [9, 22]

$$
\begin{array}{r}
\left(T+V+2 V_{H}-\mu\right) u_{j}-V_{H} v_{j}=E_{j} u_{j}, \\
-V_{H} u_{j}+\left(T+V+2 V_{H}-\mu\right) v_{j}=-E_{j} v_{j},
\end{array}
$$

where the condensate density plays the role of a nonuniform potential through the Hartree contribution $V_{H}$. (The similar coupled equations for a superconductor are usually known as the Bogoliubov-de Gennes equations 23, 24.) The associated eigenvalues $E_{j}$ determine the quasiparticle energies in the presence of the nonuniform condensate, analogous to the Bogoliubov energies in Eq. (3) for plane waves.

An equivalent approach is to introduce hermitian small-amplitude hydrodynamic operators [25, 26]

$$
\begin{array}{r}
\rho(\mathbf{r})=\sqrt{n_{0}(\mathbf{r})}\left[\phi(\mathbf{r})+\phi^{\dagger}(\mathbf{r})\right], \\
\Phi(\mathbf{r})=\frac{\hbar}{2 m i \sqrt{n_{0}(\mathbf{r})}}\left[\phi(\mathbf{r})-\phi^{\dagger}(\mathbf{r})\right]
\end{array}
$$

for a density fluctuation and a velocity potential fluctuation at the point $\mathbf{r}$ [the associated velocity is sim- ply $\mathbf{v}(\mathbf{r})=\nabla \Phi(\mathbf{r})]$. These hydrodynamic operators are merely linear combinations of the noncondensate field operators with known nonuniform coefficients, and the corresponding normal-mode amplitudes are similarly linear combinations of the Bogoliubov amplitudes. In the present case of a stationary condensate (the generalization to a condensate with a persistent current is straightforward 250), the hydrodynamic operators obey the coupled linear equations

$$
\begin{gathered}
\frac{\partial \rho}{\partial t}+\nabla \cdot\left(n_{0} \nabla \Phi\right)=0 \\
\frac{\partial \Phi}{\partial t}+\frac{4 \pi a \hbar^{2}}{m^{2}} \rho+\frac{\hbar^{2}}{4 m^{2} n_{0}}\left[\nabla \cdot\left(\rho \frac{\nabla n_{0}}{n_{0}}\right)-\nabla^{2} \rho\right]=0
\end{gathered}
$$

The first is the familiar linearized equation of particle conservation, and the second is a linearization of the classical Bernoulli's theorem for a compressible irrotational isentropic fluid obtained from the appropriate quantum energy-density functional [25]. The corresponding hydrodynamic amplitudes obey similar coupled eigenvalue equations that determine the normal-mode frequencies $\omega_{j}=E_{j} / \hbar$.

The standard theory of linear response 27] shows that the density-density correlation function characterizes the response of the condensate to a weak external perturbation that couples to the density (experimentally, this coupling can be obtained for a trapped Bose gas by modulating the curvature of the external trap [28, 29]). Since the density operator is here merely a linear combination of the original particle operators (this special form reflects the presence of a macroscopic Bose condensate, and the situation in a Fermi gas is very different), the resonant frequencies of the density-density correlation function are necessarily the same as those of the Bogoliubov equations, which provides an experimental method to determine the quasiparticle eigenvalues $E_{j}$.

If treated exactly, the Bogoliubov and the hydrodynamic descriptions both yield the same physical information about the eigenvalues and eigenfunctions. The former relies on a quantum formalism with simple boundary conditions as $r \rightarrow \infty$, and the condensate density appears solely as a multiplicative coefficient. In contrast, the latter involves various spatial derivatives of the condensate density, but it offers the advantage of physical intuition (at least from a classical perspective), for the hydrodynamic amplitudes are like the normal modes of a selfgravitating nonuniform star. Indeed, the recent field of "helioseismology" uses the observed normal modes of the sun to infer the (otherwise invisible) behav- 
ior below the solar surface; similar studies may be applicable to the trapped alkali Bose condensates.

\section{RECENT THEORETICAL STUDIES}

The last year has seen the appearance of many theoretical papers and preprints on various aspects Bose condensation in dilute trapped gases [30]; the present section can only summarize a few selected studies that illustrate the recent trends.

\subsection{Stationary condensate}

The GP equation (14) for a spherical static Bose condensate in an isotropic harmonic trap reduces to a nonlinear ordinary differential equation that can be solved in many ways, and the generalization to anisotropic traps is not difficult. A collaborative group from Georgia Southern/NIST/Oxford 31, 32, 33. has performed extensive numerical studies of the radial condensate wave function (see also (34), including the dependence on the dimensionless parameter $N a / d_{0}$ for both positive and negative values (the latter corresponds to an attractive interaction). In addition, analytical studies have treated the same problem variationally [35, 36], using trial functions that interpolate smoothly between the (ideal) Gaussian limit (for $N a / d_{0} \ll 1$ ) and the dilute (TF) $\operatorname{limit}\left(N a / d_{0} \gg 1\right)$.

Usually, the most relevant physical limit is $N a / d_{0} \gg 1$, when the TF approximation describes the spatial variation of the condensate density except in a thin layer near the surface, where the condensate kinetic energy becomes significant. Dalfovo, Pitaevskii, and Stringari have recently studied this problem analytically using boundary-layer techniques 37.

\subsection{Rotating condensate (vortices)}

The existence and behavior of vortices in rotating superfluid ${ }^{4}$ He has long been studied (see, for example, 38, 39). In particular, the superfluid velocity $\mathbf{v}_{s}(\mathbf{r})$ is proportional to the gradient of the phase of the condensate wave function. As a result, the superflow is irrotational "almost" everywhere except in discrete singular regions of dimension $\sim \xi$ (the nodes of $\Psi$ ) where the phase is undefined; superfluid vorticity (namely, the regions where $\nabla \times \mathbf{v}_{s} \neq 0$ ) is localized in the cores of the superfluid vortices. In addition, $\Psi$ is single-valued whenever its coordinate $\mathbf{r}$ traverses a closed path in the fluid, which ensures the quantization of circulation $\kappa$ in units of $h / m$.

The simplest system for liquid ${ }^{4} \mathrm{He}$ is a long circular cylindrical container with radius $R$ that can rotate about its symmetry axis. For small angular velocity $\Omega$, the fluid remains at rest with no vortices, but, at a critical value $\Omega_{c 1} \sim\left(\hbar / m R^{2}\right) \ln (R / \xi)$, it becomes favorable to create one singly quantized vortex line on the axis of the cylinder. Similar questions have been considered for axisymmetric harmonic traps in the context of Bose condensed alkali atoms 20, 33, 34, where, for current experiments on ${ }^{87} \mathrm{Rb}$, the critical angular velocity should be $\Omega_{c 1} \sim 50 \mathrm{rad} / \mathrm{s}$.

\subsection{Excited states}

As noted in the paragraph below Eq. (24), the linearized normal modes of the condensate characterize both its small-amplitude dynamics and its linear response to a weak external perturbation that couples to the particle density. As a result, considerable theoretical effort has been devoted to the calculation of these normal modes, both numerically 40 and analytically in the TF limit of a large condensate 41]. For an axisymmetric trap, the eigenstates can be labeled with the azimuthal quantum number $m$. In recent experiments [28, 29], the trap curvature was modulated at a prescribed external frequency, and the observed resonant behavior measures the appropriate eigenfrequencies. Detailed comparison between theory and experiment yields good agreement for the lowest states with $m=0$ and $m=2$.

\section{SOME OPEN QUESTIONS}

This field is developing rapidly, so that any selection of open questions is somewhat arbitrary (and sure soon to be irrelevant). Nevertheless, the following few topics are likely to remain of interest for at least the immediate future.

\subsection{Normal modes of a large condensate}

In the limit of a large condensate $\left(\mathrm{Na} / \mathrm{d}_{0} \gg 1\right)$, the TF approximation for the condensate density holds apart from the surface region. An incompressible spherical fluid has surface waves as its lowest normal modes, but it is less clear what happens for a dilute nonuniform Bose gas. In principle, numerical analysis can yield all the normal modes for a particular configuration, but analytical results would be helpful to gain some insight into the relevance of various physical parameters and the interplay between bulk modes and surface modes.

\subsection{Axisymmetric rotating condensate}

From an experimental viewpoint, the simplest way to create vortex lines in liquid ${ }^{4} \mathrm{He}$ is to cool the sample while it rotates, so that the onset of the superfluid state occurs at fixed $\Omega$. This method works even for a circular cylindrical container, because the 
rotating walls entrain the viscous normal fluid above $T_{\lambda}$, thus creating the superfluid in a state of rotation.

An alternative procedure is to cool a stationary container of ${ }^{4} \mathrm{He}$ below $T_{\lambda}$ and then spin it up to some final angular velocity $\Omega$. For $\Omega \lesssim \Omega_{c 1}$, the superfluid typically remains at rest, but for $\Omega \gtrsim \Omega_{c 1}$, the inevitable surface roughness of the rotating wall apparently initiates the formation of a vortex. Unfortunately, the detailed mechanism for vortex creation remains unclear, and the observed hysteresis implies significant metastability 42.

In liquid ${ }^{4} \mathrm{He}$, only singly quantized vortices are expected theoretically, in agreement with many different experimental observations [39, 42]. Imaging the vortices in rotating superfluid ${ }^{4}$ He remained elusive for many years, in contrast to the corresponding success with quantized flux lines in type-II superconductors [17]. Nevertheless, clever use of ion trapping on the vortex cores 38, 39 eventually yielded remarkably explicit images of the vortex positions 43. 44, which are really just the nodes in the condensate wave function. Similar explicit images of vortices in dilute trapped alkali Bose condensates would be very desirable (it is not even obvious that only singly quantized vortex lines will occur).

\subsection{Nonaxisymmetric rotating condensate}

As noted above, spinning up a long circular cylinder of superfluid ${ }^{4} \mathrm{He}$ from rest frequently involves hysteresis, and it is not clear how a similar procedure for condensed alkali atoms in an axisymmetric trap can succeed in creating vortices (in the absence of physical boundaries, what does it mean to rotate the trap?). One obvious possibility is to use an asymmetric container (for example, different oscillator lengths $d_{x}$ and $d_{y}$ in the $x y$ plane, with the harmonic trap rotating about the $z$ axis).

A similar question has been treated theoretically for an incompressible superfluid in a long cylinder with either elliptical or rectangular profile [45]. The principal new feature is that the rotating walls impel the superfluid into irrotational motion even in the absence of vortices. Indeed, for an elliptical profile with semiaxes $a$ and $b$, the resulting induced angular momentum is reduced relative to that for solid-body rotation by a factor $\left(a^{2}-b^{2}\right)^{2} /\left(a^{2}+b^{2}\right)^{2}$ 45, which vanishes for a circular profile but is otherwise positive and less than one. In addition, the critical angular velocity $\Omega_{c 1}$ for the onset of vortex formation is increased relative to that for a circular cylinder for the following physical reason. It is easy to show that solid-body rotation $\boldsymbol{\Omega} \times \mathbf{r}$ is the true equilibrium of an unconstrained rotating incompressible fluid in a container, but such a configuration is forbidden to a superfluid because $\mathbf{v}_{s}$ must remain irrotational almost everywhere (recall that solid-body rotation has uniform nonzero vorticity $2 \boldsymbol{\Omega}$ ). A superfluid in a rotating circular cylinder has no purely irrotational motion and can mimic solid-body rotation only by creating singly quantized vortices; in contrast, a rotating elliptic cylinder does have irrotational flow that already acts somewhat like solid-body rotation, reducing the need to create one or more discrete vortices. Careful experiments confirm this prediction in considerable detail 46.

\subsection{Excited states of a vortex}

A classical rectilinear vortex with circulation $\kappa$ in an incompressible fluid has oscillatory normal modes that propagate along (and are confined to) the axis, with a long-wavelength dispersion relation $\omega \approx\left(\kappa k^{2} / 4 \pi\right) \ln (1 / k \xi)$, where $k$ is the axial wavenumber and $\xi$ is the core size (assuming $k \xi \ll 1$ ) 38, 39. Similar vortex waves occur for a vortex in an unbounded dilute Bose condensate [9, 47], where $\kappa=h / m$. In addition, the phonon-like modes of a uniform Bose gas are modified by the presence of a vortex, leading to asymptotic phase shifts. The resulting phonon-vortex scattering contributes to the mutual friction, as observed in rotating superfluid ${ }^{4}$ He through an excess attenuation of second sound relative to that for stationary superfluid [38, 39].

The situation for one or more vortices in a trapped dilute Bose condensate will necessarily differ considerably from that in an unbounded medium. As one simple example, the ratio of the condensate radius $R$ to the vortex core $\xi$ is typically $\sim 10$, so that only relatively few vortices can form before the cores overlap and the system becomes "normal" (a finite-size analog of the destruction of superconductivity at the upper critical field $H_{c 2}$ [17]). A fundamental question concerns the various possible normal modes that can occur for vortex lines in these relatively small dilute trapped systems. Although there will certainly be phonon-like modes that are scattered by the vortices, it is less obvious that such a system can support an analog of the bound vortexwave modes.

\section{REFERENCES}

[1] F. London, Superfluids (Dover, NY, 1964), Vol. II, provides a comprehensive account of the experimental and theoretical situation as of 1954 , with very complete references.

[2] The experimental summary of J. Wilks, The 
Properties of Liquid and Solid Helium (Clarendon Press, Oxford, 1967), Chaps. 1-8, remains remarkably up-to-date.

[3] L. D. Landau and E. M. Lifshitz Fluid Mechanics (Pergamon, Oxford, 1987), 2nd ed., Chap. XVI summarizes Landau's two-fluid hydrodynamics.

[4] See also A. L. Fetter and J. D. Walecka Quantum Theory of Many-Particle Systems (McGraw-Hill, NY, 1971), Sec. 54.

[5] N. N. Bogoliubov, J. Phys. (USSR) 11, 23 (1947).

[6] Bogoliubov's seminal paper is reprinted in D. Pines, The Many-Body Problem (Benjamin, NY 1961) along with a brief historical summary and reprints of many of the central subsequent papers, specifically those by Lee, Huang, and Yang, by Beliaev, and by Hugenholtz and Pines.

[7] See also Ref. [ 4 , Chap. 6 and Sec. 35.

[8] E. P. Gross, Nuovo Cimento 20, 454 (1961); J. Math. Phys. 46, 137 (1963).

[9] L. P. Pitaevskii, Zh. Eksp. Teor. Fiz. 40, 646 (1961); Sov. Phys.-JETP 13, 451 (1961).

[10] See also Ref. 四, Sec. 55.

[11] M. H. Anderson, J. R. Ensher, M. R. Matthews, C. E. Wieman, and E. A. Cornell, Science 269, 198 (1995).

[12] C. C. Bradley, C. A. Sackett, J. J. Tollett, and R. G. Hulet, Phy. Rev. Lett. 75, 1687 (1995).

[13] K. B. Davis, M.-O. Mewes, M. R. Andrews, N. J. van Druten, D. S. Durfee, D. M. Kurn, and W. Ketterle, Phys. Rev. Lett. 75, 3969 (1995).

[14] M.-O. Mewes, M. R. Andrews, N. J. van Druten, D. M. Kurn, D. S. Durfee, and W. Ketterle, Phys. Rev. Lett. 77, 416 (1996).

[15] M. Tinkham, Introduction to Superconductivity (McGraw-Hill, NY, 1996), 2nd ed., Sec. 3.5.

[16] V. L. Ginzburg and L. P. Pitaevskii, Zh. Eksp. Teor. Fiz. 34, 1240 (1958); Sov. Phys.-JETP 7, 858 (1958).

[17] See, for example, Ref. [1], Sec. 50 and Ref. [15], Chaps. 4 and 5.
[18] V. V. Goldman, I. F. Silvera, and A. J. Leggett, Phys. Rev. B 24, 2879 (1981).

[19] D. A. Huse and E. D. Siggia, J. Low Temp. Phys. 46, 137 (1982).

[20] G. Baym and C. J. Pethick, Phys. Rev. Lett. 76, 6 (1996).

[21] See, for example, Ref. 伍, Sec. 56.

[22] A. L. Fetter, Ann. Phys. (N.Y.) 70, 67 (1972).

[23] P. G. de Gennes, Superconductivity of Metals and Alloys (Benjamin, NY, 1966), Chap. 5.

[24] See also Ref. [15], Sec. 10.1.

[25] A. L. Fetter, Phys. Rev. A 53, 4245 (1996).

[26] W.-C. Wu and A. Griffin, cond-mat/9606076 (unpublished).

[27] See, for example, Ref. [4], Chaps. 5 and 9.

[28] D. S. Jin, J. R. Ensher, M. R. Matthews, C. E. Wieman, and E. A. Cornell, Phys. Rev. Lett. 77, 420 (1996)

[29] M.-O. Mewes, M. R. Andrews, N. J. van Druten, D. M. Kurn, D. S. Durfee, C. G. Townsend, and W. Ketterle, Phys. Rev. Lett. 77, 988 (1996).

[30] See http://amo.phy.gasou.edu/bec.html/ for an updated bibliography on Bose-Einstein condensation (and other relevant information).

[31] M. Edwards and K. Burnett, Phys. Rev. A 51, $1382(1995)$.

[32] P. A. Ruprecht, M. J. Holland, K. Burnett, and M. Edwards, Phys. Rev. A 51, 4704 (1995).

[33] M. Edwards, R. J. Dodd, C. W. Clark, P. A. Ruprecht, and K. Burnett, Phys. Rev. A 53, R1950 (1996).

[34] F. Dalfovo and S. Stringari, Phys. Rev. A 53, 2477 (1996).

[35] K. N. Ilinski and A. Moroz, cond-mat/9512155 (to appear in J. Res. NIST).

[36] A. L. Fetter, cond-mat/9607177 (unpublished).

[37] F. Dalfovo, L. P. Pitaevskii, and S. Stringari, cond-mat/9604069 (unpublished). 
[38] A. L. Fetter, in The Physics of Liquid and Solid Helium, edited by K. H. Bennemann and J. B. Ketterson (Wiley, NY, 1976), Part I, p. 207.

[39] R. J. Donnelly, Quantized Vortices in Helium II (Cambridge Press, Cambridge, 1991).

[40] M. Edwards, P. A. Ruprecht, K. Burnett, R. J. Dodd, and C. W. Clark, Phys. Rev. Lett. 77, 1671 (1996).

[41] S. Stringari, Phys. Rev. Lett. 77, 2360 (1996).

[42] R. E. Packard and T. M. Sanders, Jr., Phys. Rev. Lett. 22, 823 (1969); Phys. Rev. A 6, 799 (1972).

[43] E. J. Yarmchuk, M. J. V. Gordon, and R. E. Packard, Phys. Rev. Lett. 43, 214 (1979).

[44] E. J. Yarmchuk and R. E. Packard, J. Low Temp. Phys. 46, 479 (1982).

[45] A. L. Fetter, J. Low Temp. Phys. 16, 533 (1974).

[46] K. DeConde and R. E. Packard, Phys. Rev. Lett. 35, 732 (1975).

[47] A. L. Fetter, Phys. Rev. 138, A709 (1965). 